5. T. P. Kirkman, Schoolgirl problem, The Lady's and Gentleman's Diary (1950).

6. F. N. Cole, Kirkman parades, Bull. Amer. Math. Soc. vol. 28 (1922) pp. 435437.

7. A. Sade, An omission in Norton's list of $7 \times 7$ squares, Ann. Math. Statist. vol. 22 (1951) pp. 306-307.

SYRACUSE UNIVERSITY

\title{
UNIQUENESS OF THE PROJECTIVE PLANE WITH 57 POINTS
}

MARSHALL HALL, JR.

1. Introduction. In the preceding paper, W. A. Pierce has given a new proof that Fano's configuration cannot exist in a projective plane with 8 points per line. In this paper, I shall use his result to give a proof of the theorem that any projective plane with 8 points per line is Desarguesian. (For historical notes, see the preceding paper.)

2. The proof. If $A_{1}, A_{2}, A_{3}, A_{4}$ form a quadrangle whose diagonal points are not collinear, then we have the following configuration:

$L_{1}: \quad A_{1} A_{2} B_{1} C_{5}$

$L_{2}: A_{1} A_{3} B_{2} C_{3}$

$L_{3}: A_{1} A_{4} B_{3} C_{1}$

$L_{4}: \quad A_{2} A_{3} B_{3} C_{2}$

$L_{5}: A_{2} A_{4} B_{2} C_{4}$

$L_{6}: A_{3} A_{4} B_{1} C_{6}$

$M_{1}: \quad B_{1} B_{2} C_{1} C_{2}$

$M_{2}: \quad B_{1} B_{8} C_{8} C_{4}$

$M_{8}: \quad B_{2} B_{8} C_{6} C_{6}$

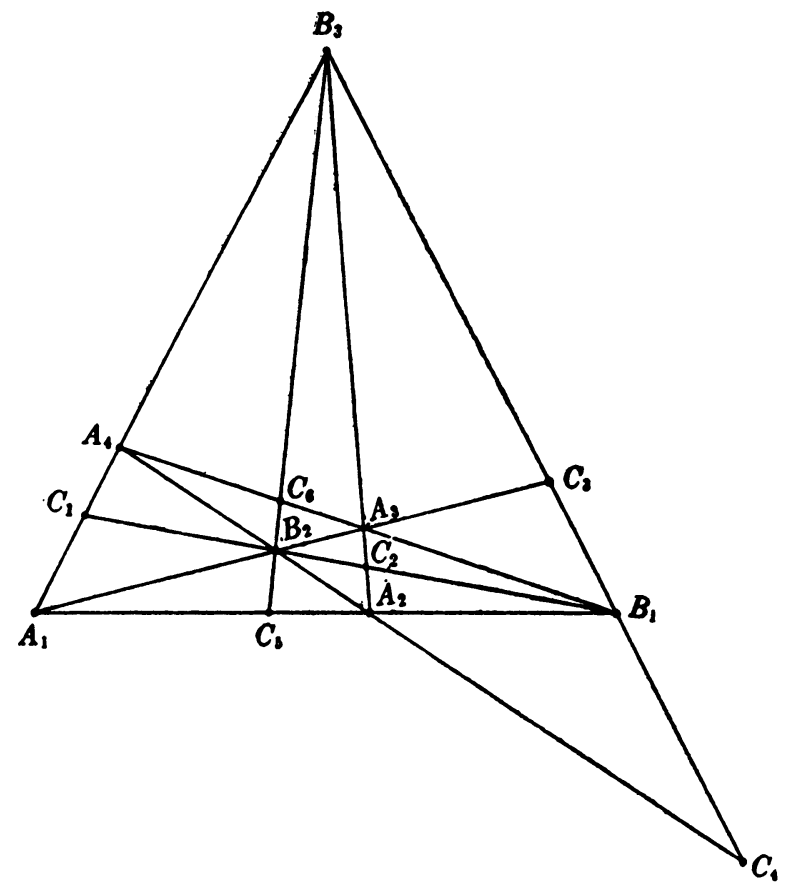

Presented to the Society, April 24, 1953; received by the editors February 17, 1953. 
No further incidences between these points and lines are possible (e.g., $C_{5}$ could not lie on $L_{4}$ ). The 9 lines intersect one another as indicated and further points on these lines are all distinct.

Let us designate further points on lines $L$ as $D$ 's, further points on lines $M$ as $E$ 's and all remaining points as $F$ 's. The following indicates all possible line patterns:

\begin{tabular}{|c|c|c|}
\hline$L: A A B C D D D D$ & $4 A$ 's & $6 L ' s$ \\
\hline$M: \quad B B C C E E E E$ & $3 B$ 's & $3 M$ 's \\
\hline$N: \quad B D D D D E F F$ & $6 C$ 's & $12 N ' s$ \\
\hline$R: C D D D D D E E$ & $24 D^{\prime} \mathrm{s}$ & \\
\hline$S: A D D D E E E F$ & $12 E^{\prime} \mathrm{s}$ & \\
\hline$T: A C D D E E F F$ & $8 F^{\prime} \mathrm{s}$ & \\
\hline$U: A C C D E F$ F $F$ & 57 & \\
\hline$V: A C C A F F F$ & & \\
\hline$W: C C D D D D E F$ & & \\
\hline$X: C C C D D D F F$ & & \\
\hline
\end{tabular}

Here, for example, a line of type $T: A C \cdots$ intersects $3 L$ 's in the $A$ and one other in the $C$; hence it must intersect the two remaining in $D$ 's. It intersects one $M$ in the $C$ and the 2 others in $E$ 's. The remaining two points will be $F$ 's. A line of type $Y$, not through any $A, B$, or $C$, would have to contain $6 D$ 's and $3 E$ 's but this is impossible.

We shall use the letters $R, S, T, U, V, X$ to stand also for the number of lines of its type. Thus

$$
R+S+T+U+V+W+X=36 \text {. }
$$

Counting $A$ 's and recalling that there are 8 lines through each point,

$$
12+S+T+U+V=32 .
$$

Counting pairs $A C$,

$$
12+T+2 U+3 V=24
$$

Counting $C^{\prime}$ s,

$$
12+R+T+2 U+3 V+2 W+3 X=48 .
$$

Counting pairs $C C$,

$$
3+U+3 V+W+3 X=15 .
$$

Further relations yield nothing independent. Here 


$$
\begin{aligned}
& R=12-U-3 V \\
& S=8+U+2 V \\
& T=12-2 U-3 V \\
& W=\quad 2 U+6 V \\
& X=4-U-3 V .
\end{aligned}
$$

From the $X$ value we see that $V=0$ or 1 . I will first eliminate the possibility $V=1$.

We consider the points $C$ in more detail and note that a line $R$ and a line $V$ intersect in a point $C$.

An individual $C$ occurs on $1 L$ and on $1 M$. Let it also occur on $r R^{\prime} \mathrm{s}, t T$ 's, $u U^{\prime} \mathrm{s}, v V^{\prime} \mathrm{s}, w W^{\prime} \mathrm{s}$, and $x X^{\prime}$ 's. Counting its 8 occurrences

$$
2+r+t+u+v+w+x=8 \text {. }
$$

Since it must join each of the $4 A$ 's,

$$
2+t+u+v=4 \text {. }
$$

$C C$ (joining the remaining $C^{\prime} \mathrm{s}$ )

$$
1+u+2 v+w+2 x=5 .
$$

Other relations yield nothing new. Here

$$
\begin{aligned}
r & =u+2 v+x, \\
t & =2-u-v, \\
w & =4-u-2 v-2 x .
\end{aligned}
$$

The solutions of these equations are

$\begin{array}{lllllll}r & t & u & 0 & w & x & \\ 4 & 0 & 0 & 2 & 0 & 0 & \\ 3 & 0 & 1 & 1 & 1 & 0 & c_{1} \\ 3 & 1 & 0 & 1 & 0 & 1 & c_{2} \\ 2 & 1 & 0 & 1 & 2 & 0 & c_{8} \\ 2 & 2 & 0 & 0 & 0 & 2 & c_{4} \\ 1 & 2 & 0 & 0 & 2 & 1 & c_{8} \\ 0 & 2 & 0 & 0 & 4 & 0 & c_{8} \\ 2 & 1 & 1 & 0 & 1 & 1 & c_{7} \\ 1 & 1 & 1 & 0 & 3 & 0 & c_{8}\end{array}$




$\begin{array}{lllllll}3 & 0 & 2 & 0 & 0 & 1 & c_{0} \\ 2 & 0 & 2 & 0 & 2 & 0 & c_{10}\end{array}$

When $V=1$ then $U=0$ or 1 .

Case $(\alpha) . R=9, S=10, T=9, U=0, V=1, W=6, X=1$.

Since $U=0, c_{1}=c_{7}=c_{8}=c_{9}=c_{10}=0$.

(No $C^{\prime}$ 's of these types.) Also $c_{4}=0$ (from $X=1$ ).

From $V=1, c_{2}+c_{8}=3$.

$R V$ intersections $3 c_{2}+2 c_{3}=9$.

Hence $c_{2}=3, c_{3}=0$. But with $c_{2}=3$ we have $3 V X$ intersections conflicting with $V=1, X=1$. Hence Case $(\alpha)$ is out.

Case $(\beta) . R=8, S=11, T=7, U=1, V=1, W=8, X=0$.

From $X=0, c_{2}=c_{4}=c_{8}=c_{7}=c_{9}=0$.

From $V=1, c_{1}+c_{3}=3$.

$R V=8,3 c_{1}+2 c_{3}=8$. Hence $c_{1}=2, c_{3}=1$. But $c_{1}=2$ yields $2 U V$ intersections contrary to $U=1, V=1$. This is a conflict.

Hence $V=0$.

With $V=0$, consider a point $A$ on $3 L$ 's, $s S^{\prime}$ s, $t T$ 's, and $u U$ 's.

8 occurrences

$A C$

$A D$

$$
\begin{aligned}
3+s+t+u & =8 . \\
3+t+2 u & =6 . \\
12+3 s+2 t & =24 .
\end{aligned}
$$

Here $s=2, t=3, u=0$.

Since there is no $A$ on any $U$ we must have $U=0$.

With $U=0, V=0$

$$
\begin{array}{rr}
R=12 \\
S=8 \\
T=12 \\
W=0 \\
X=4 .
\end{array}
$$

With the $4 X$ lines we must have one or the other of the following:

$$
\begin{aligned}
& \begin{array}{lll}
C_{1} & C_{3} & C_{6}
\end{array} \\
& \begin{array}{lll}
C_{1} & C_{3} & C_{8}
\end{array} \\
& \begin{array}{llll}
X^{\prime} \mathrm{s} & C_{1} & C_{4} & C_{6} \\
& C_{2} & C_{3} & C_{6}
\end{array} \\
& \text { or } X^{\prime} \text { s } \\
& \begin{array}{llll}
C_{1} & C_{4} & C_{6}
\end{array} \\
& \begin{array}{lll}
C_{2} & C_{3} & C_{6}
\end{array} \\
& C_{2} C_{4} C_{5} \\
& C_{2} C_{4} C_{6} \text {. }
\end{aligned}
$$

But $C_{1}, C_{3}, C_{5}$ are the diagonal points of the quadrilateral $A_{1}, B_{1}, B_{2}$, $B_{3}$, and if they were collinear, we would have a Fano configuration. 
Since this has been ruled out, the second alternative must hold. But these are the configurations of Moufang's Theorem $\mathrm{D}_{8}$ ( “A-Netz") [1], and the net generated (which must be the whole plane) will be Desarguesian.

REMARK. For a geometry with 9 points on a line, the Theorem of Desargues and the Configuration of Fano imply each other. It seems plausible that the only geometry which exists is the one in which these theorems are valid. To prove this it would be sufficient to show the impossibility of a quadrangle such as that studied above whose diagonal points are not collinear. With 9 points on a line we must also admit lines of a type $Y$ with $6 D$ 's and $3 E$ 's. It can be shown that there can be no line of type $V$. But numbers satisfying all incidence requirements have been found. Thus further methods will be needed if the techniques of this paper can be extended to prove the uniqueness of the geometry with 73 points.

\section{BIBLIOGRAPHY}

(See also the preceding paper)

1. R. Moufang, Zur Struktur der projektiven Geometrie der Ebene, Math. Ann. vol. 105 (1931) pp. 536-601.

Ohio State University 